\title{
Obtención de la respuesta en frecuencia en transformadores estando en servicio
}

\author{
Obtaining the frequency response in transformers in service \\ E. Gómez-Luna ${ }^{1 *} \quad$ G. Aponte M. ${ }^{2} \quad$ J. Pleite G. ${ }^{3}$ \\ Recibido 1 de diciembre de 2014, aceptado 9 de mayo de 2016 \\ Received: December 1, 2014 Accepted: May9, 2016
}

\begin{abstract}
RESUMEN
Este artículo presenta la obtención de la respuesta en frecuencia en transformadores de distribución, estando en servicio (prueba FRA on-line), mediante la inyección de señales controladas y empleando un novedoso algoritmo para el análisis de señales transitorias.

Se usó un sistema hardware para inyectar los pulsos controlados sobre la onda de $60 \mathrm{~Hz}$, el cual permitía cambiar la amplitud, el ancho y la fase del pulso generado sobre la onda de $60 \mathrm{~Hz}$, asegurando un alto contenido espectral y un control del mismo.

De acuerdo con los resultados obtenidos se pudo observar cómo el nuevo método propuesto ofrecía mayor confiabilidad en los resultados, por lo que, el poder llevar a cabo un monitoreo on-line confiable en transformadores por medio de la técnica FRA es algo innovador y de mucho interés para las empresas del sector eléctrico, debido a que en el momento de realizar la prueba FRA no es necesario interrumpir el suministro de energía eléctrica, eliminado los altos costos que se generan al tener que sacar el transformador de servicio del sistema de potencia, por otro lado, la importancia del sistema implementado es poder supervisar el transformador desde una red inteligente (Smart Grid) con el fin de tomar decisiones oportunas del estado real de operación basado en la condición del equipo.

La metodología desarrollada se aplicó a transformadores piloto y a transformadores de una red de distribución del sistema de potencia con el objeto de validar el método propuesto.
\end{abstract}

Palabras clave: Respuesta en frecuencia on-line, transformadores, procesamiento de señal, monitoreo on-line, señales transitorias.

\begin{abstract}
This article presents obtaining the frequency response in transformers, in service (on-line FRA test), through the injection of controlled signals and employing a new algorithm for the analysis of transitory signals. A system hardware was used for the injection of controlled pulses on the $60 \mathrm{~Hz}$; this system can inject signals and change its amplitude, width and phase, ensuring a high spectral content and control of it. According to the results, it was observed how the new method provided greater confidence in the results, so the place to bring a reliable on-line transformer monitoring through the FRA technique is innovative and much interest to electric utilities, because now in the FRA test will be not necessary to interrupt the power supply, eliminating the high costs generated by having to de energized the transformer, the importance of the implemented system is that is capable to monitor the transformer from a smart grid in order to make timely decisions in the actual state of operation based on the equipment condition.
\end{abstract}

Keywords: On-line frequency response, transformers, signal processing, on-line monitoring, transitory signal.

1 Grupo de Investigación GITICAP. Potencia y Tecnologías Incorporadas S.A. Santiago de Cali, Colombia. Carrera 56, 2-50, Cali, Colombia. E-mail: eduardo.gomez@pti-sa.com.co

2 Grupo de Investigación en Alta Tensión. Universidad del Valle. Santiago de Cali, Colombia. Calle 13 \#100-00. Edificio 356, AA 25360. E-mail: guillermo.aponte@ correounivalle.edu.co

3 Departamento de Tecnología Electrónica. Universidad Carlos III de Madrid. Madrid, España. Avenida de la Universidad 30, 28911. Leganés, España. E-mail: pleite@ing.uc3m.es

* Autor de correspondencia 


\section{INTRODUCCIÓN}

El análisis de la respuesta en frecuencia (FRA, Frequency Response Analysis) es una técnica para la evaluación del estado de los transformadores, utilizada a nivel mundial como complemento y apoyo de otras técnicas de diagnóstico. Es especialmente utilizada para la detección de posibles problemas mecánicos, tales como desplazamientos o deformaciones en los arrollamientos o chapas del núcleo, las que son muy difíciles de localizar con otros métodos [1-4].

Hasta el momento la técnica de respuesta en frecuencia en los transformadores tiene dos grandes limitantes: La interpretación de las respuestas medidas para la obtención de un diagnóstico confiable necesita de investigación más avanzada dado que aún no se toman decisiones a partir de FRA, es una técnica complementaria, falta por investigar en relación con la distribución de los diferentes efectos que se tiene del transformador sobre la respuesta, además la cuantificación de los efectos de daño y, la segunda, es necesario desconectar del sistema de potencia el transformador para realizar la prueba (medida off-line).

En relación con el primer punto, hasta el momento se han desarrollado avances para la interpretación de las curvas obtenidas off-line, principalmente orientados al uso de modelos del transformador que sean capaces de reproducir el mismo comportamiento en frecuencia del sistema medido (transformador) $[1,5-8]$.

La segunda limitación radica en el caso que exista alguna sospecha de fallo por salidas inoportunas del sistema de potencia por lo que es necesario realizar algunas pruebas para verificar que el transformador no ha sufrido cambios internos severos, como es el caso de la técnica FRA, motivo por el que es necesario la desconexión del transformador para la realización de dicha prueba, generando altos costos y disminuyendo la confiabilidad del suministro de energía eléctrica. Por tanto, la medida on-line del estado del transformador mediante la técnica de respuesta en frecuencia presentaría ventajas sustanciales, lo que ha generado un interés notable entre el sector productivo y la comunidad científica [9-13].

Con el objeto de definir el procedimiento estándar de la prueba FRA con el transformador fuera de línea han sido presentados documentos como: la Norma de la República popular de China en el 2005 [14], la guía del CIGRE para la realización de la prueba FRA, en el 2008 [15], seguido a esto se manifiestan los comités de normatividad internacional como la IEEE [1] e IEC [2] publicando la normatividad vigente para realizar la prueba FRA.

Los grandes avances obtenidos hasta el momento en relación con la técnica FRA estando el transformador fuera de servicio (off-line) han sido una motivación para la realización de la presente investigación, dado que se pueden reducir los costos de mantenimiento y aumentar la confiabilidad del suministro de energía al momento de realizar la prueba FRA con el transformador en servicio.

Este trabajo de investigación se enfoca en la segunda limitación, la que consiste en proponer una alternativa para la medida y análisis de la prueba FRA on-line en transformadores, con el fin de incluir el sistema de medida en un sistema de Smart Grid en el sistema de potencia.

El artículo está organizado de la siguiente manera, inicialmente se presenta la metodología propuesta para la aplicación FRA on-line, los equipos empleados para las pruebas y la descripción de cada una de las etapas propuestas; luego se muestran las pruebas realizadas en laboratorio y campo, presentando los resultados obtenidos y el respectivo análisis.Por último se presentan las conclusiones sobre el trabajo de investigación desarrollado, los aportes y productos obtenidos.

\section{METODOLOGÍA IFRA ON-LINE EN TRANSFORMADORES}

En la Figura 1 se muestra el diagrama a seguir para llevar a cabo la metodología propuesta en el trabajo de investigación para obtener FRA on-line.

De acuerdo con la Figura 1 se describen cada una de las etapas en relación con la inyección de la señal de excitación, su medida al igual que el procesamiento y el análisis de resultados, por lo que se han clasificado las señales de entrada para IFRA on-line en dos tipos: Las señales no controladas y las señales controladas. El método propuesto está compuesto por tres etapas, las que fueron aportes del trabajo de investigación 


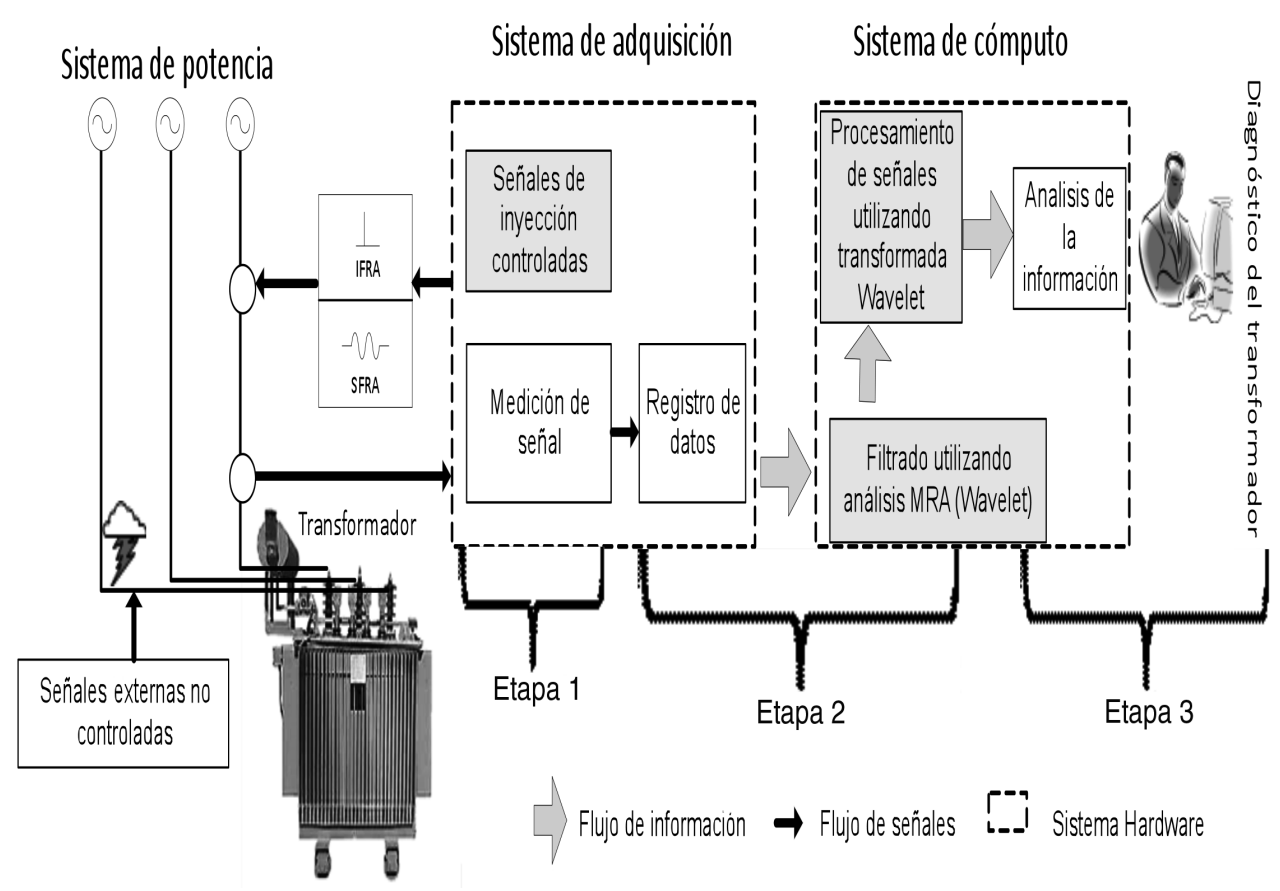

Figura 1. Definición de las etapas para llevar a cabo IFRA on-line.

[13] desarrollado y las cuales se describen a continuación.

\section{Etapa 1: Inyección y medida de la señal}

La etapa 1 se refiere a la inyección de la señal de excitación y su medida junto con la señal de salida. Para el sistema de inyección se diseñó un circuito electrónico dedicado específicamente a inyectar pulsos controlados sobre la onda de $60 \mathrm{~Hz}$.

El sistema de inyección permite cambiar la amplitud, el ancho y la fase del pulso generado sobre la onda de $60 \mathrm{~Hz}$, asegurando que posee un alto contenido espectral y un control del mismo.

Los pulsos enviados al transformador bajo ensayo se realizaron por medio de un capacitor de alto voltaje, el capacitor se convierte en un filtro paso alto para la señal de baja frecuencia, es decir, que la señal de $60 \mathrm{~Hz}$ se anula y solo deja pasar los pulsos de alta frecuencia al transformador bajo ensayo.

La Figura 2 presenta los pulsos inyectados sobre la señal de voltaje y la Figura 3 muestra la respuesta en la señal de corriente con una ventana de tiempo de $1 \mathrm{~ms}$.

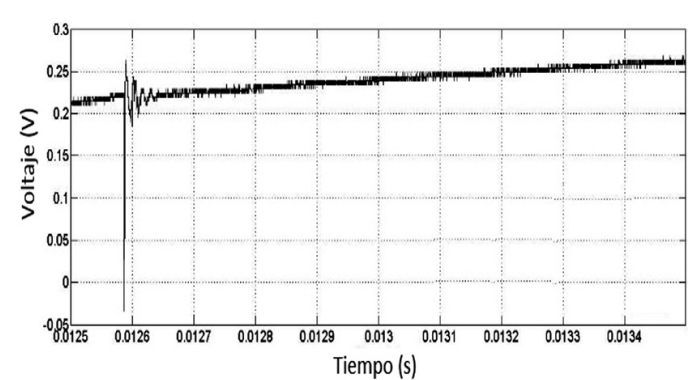

Figura 2. Pulso controlado de voltaje sobre la onda de $60 \mathrm{~Hz}$.

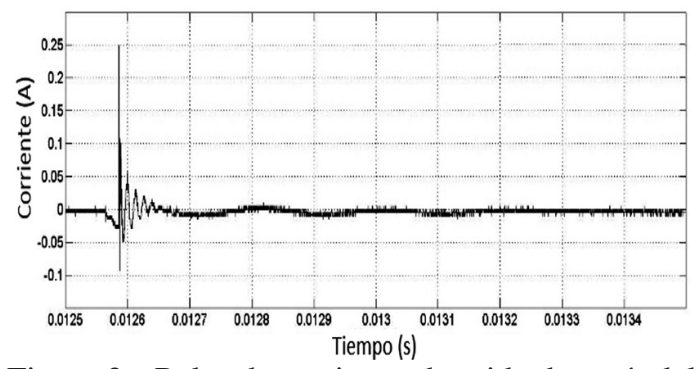

Figura 3. Pulso de corriente obtenido después del proceso de filtrado.

La Figura 4 presenta un esquema para la conexión de los equipos de medida, por uno de los lados del transformador. 


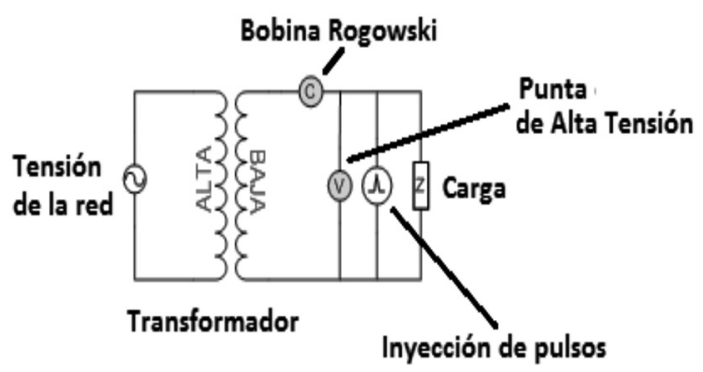

Figura 4. Diagrama esquemático para la conexión de los equipos de medida.

Para la medida de las señales de voltaje y corriente se emplearon sensores con amplios anchos de banda, puntas de alta tensión para los pulsos de voltaje y bobinas de Rogowski para la corriente, lo que permitió el registro de transitorios sin problemas de saturación magnética, teniendo en cuenta que los pulsos son de corta duración y alto contenido espectral.

\section{Etapa 2: Adquisición, filtrado y procesamiento matemático de señal}

Las señales de voltaje y corriente fueron registradas a una frecuencia de muestreo de 10 y $20 \mathrm{MS} / \mathrm{s}$ con una resolución de 12 bits, la idea era poder registrar transitorios de muy corta duración (microsegundos o nanosegundos) con el objeto de obtener un espectro en frecuencia desde los cientos de Hercios hasta 1 $\mathrm{MHz}$, rango validado para la prueba FRA según la normatividad internacional [1-2].

La Figura 5 presenta un esquema del proceso de registro de datos junto con los equipos de medida.

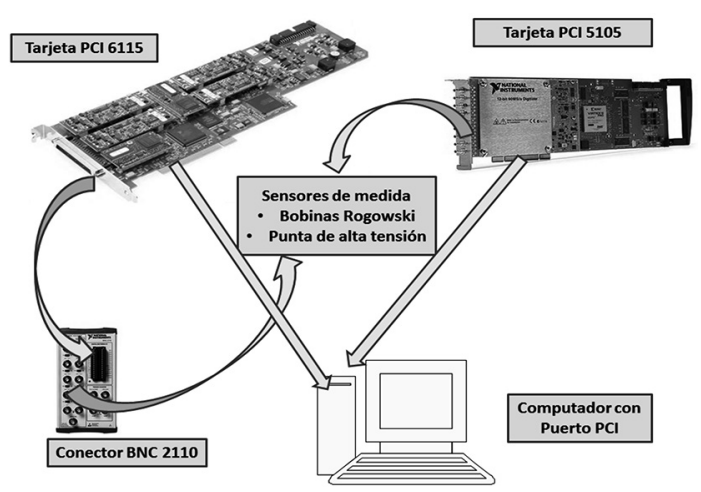

Figura 5. Tarjetas de adquisición de datos y proceso de registro de la información.
Dado que los pulsos controlados sobre la señal de voltaje (señal inyectada) y los de la señal de corriente (respuesta obtenida) viajan junto con la onda del sistema eléctrico al igual que los armónicos del sistema fue necesario modelar de manera general la suma de señales estacionarias y señales transitorias (no estacionarias) las que estaban presentes durante el proceso de medida de forma simultánea y que hace necesario discriminar.

La ecuación (1) representa el conjunto de señales involucradas en la obtención de IFRA on-line.

$$
\mathrm{S}(\mathrm{t})=T(t)+\mathrm{h}(\mathrm{t})+\mathrm{r}(\mathrm{t})
$$

Donde:

$\mathrm{T}(\mathrm{t})$ : representa la respuesta transitoria que depende directamente del comportamiento del transformador [16].

$h(t)$ : representa el conjunto de armónicos estacionarios presentes en el sistema de potencia debido a la energización, en el que el armónico fundamental es la frecuencia de red $(60 \mathrm{~Hz}$ para Colombia).

$r(t)$ : representa el ruido presente en el sistema eléctrico.

La Figura 6 presenta el esquema del tratamiento de señal que se le realizó a S(t) (ver ecuación (1)),

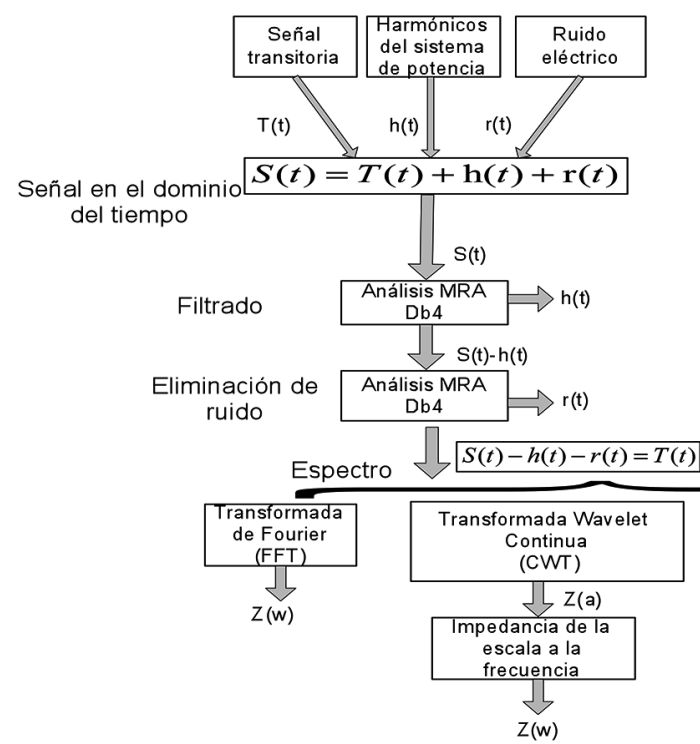

Figura 6. Diagrama de bloques del procesamiento de la señal $\mathrm{S}(\mathrm{t})$. 
con el fin de obtener el espectro en frecuencia de las señales de voltaje y corriente para obtener FRA on-line.

Para el filtrado y procesamiento de señal se usó la Transformada Wavelet la que ha sido ampliamente usada durante las dos últimas décadas como una herramienta novedosa para el filtrado, eliminación de ruido y procesamiento de señales eléctricas [13, 17-22].

En este estudio se utilizó el análisis de Multirresolución de la Transformada Wavelet Discreta (DWT), en inglés Multiresolution Analysis, MRA, desarrollado por Mallat en 1988 [23].

La Figura 7, muestra el proceso inicial de la descomposición sucesiva a la señal "S" utilizando bancos de filtros, el ancho de banda de la señal se divide en dos, haciendo uso de un filtro paso alto para obtener los detalles " $\mathrm{D}_{\mathrm{n}}$ " $\mathrm{y}$ un filtro paso bajo para las aproximaciones " $\mathrm{A}_{\mathrm{n}}$ ", en cada nivel "n" [24-25].

El filtrado implementado mediante Transformada Wavelet Discreta fue necesario para eliminar los armónicos del sistema de potencia $\mathrm{h}(\mathrm{t})$ involucrando la señal de red $(60 \mathrm{~Hz})$, tal como se explica en las Figuras 6 y 7 con el diagrama de bloques presentado, este filtrado consistió en aplicar el análisis MRA hasta un nivel de descomposición 13, usando la madre Wavelet Daubechies 4 (db4), tal como se sugiere en [24] y [25], dado que con ese nivel de descomposición se pueden eliminar los coeficientes que poseen la información de la última aproximación del proceso

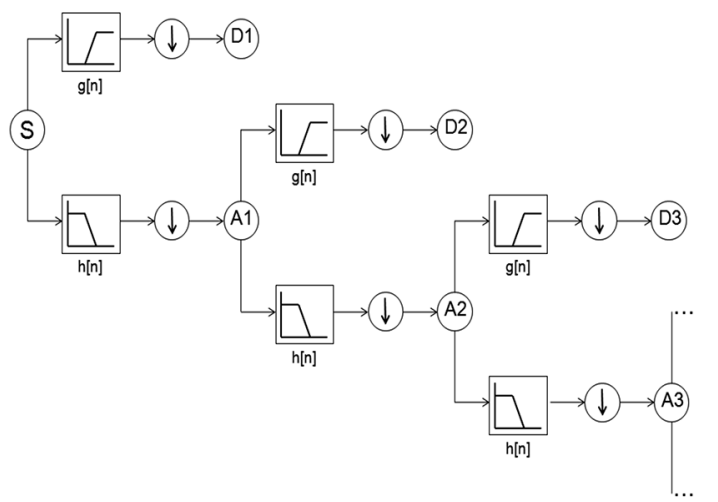

Figura 7. Árbol de descomposición de la señal S usando análisis MRA. de filtrado correspondiente a los armónicos de baja frecuencia hasta $600 \mathrm{~Hz}$ [18-23].

Para eliminar el ruido del sistema eléctrico $(\mathrm{r}(\mathrm{t})$ (ecuación (1)) se usó nuevamente la Wavelet madre $\mathrm{db} 4$, pero en el nivel 10, dado que el ruido presente en el sistema eléctrico se elimina alrededor de las bajas frecuencias como se realiza en [26] y [27].

Luego del proceso de registro, filtrado y eliminación de ruido de las señales de voltaje $\mathrm{v}(\mathrm{t})$ y corriente $\mathrm{i}(\mathrm{t})$, se realizó el procesamiento de señal usando los métodos tradicionales y la Transformada Wavelet Continua (CWT) con el objeto de determinar cuál de los métodos presentaba mejores resultados.

La Transformada Wavelet continua está definida en la ecuación (2) y a diferencia de la Transformada Discreta donde se obtienen coeficientes de detalle y aproximación solo se obtienen coeficientes para una escala dada.

$$
C(\tau, a)=\frac{1}{\sqrt{a}} \int_{-\infty}^{\infty} x(t) \overline{\psi\left(\frac{t-\tau}{a}\right)} d t
$$

Donde $\psi$ indica el complejo conjugado de la Wavelet madre, $\mathrm{x}(\mathrm{t})$ la señal a transformar, el parámetro " $a$ " provee dilatación (o escalamiento) el que varía de manera continua, el parámetro " $\tau$ " cumple la función de traslación y $\mathrm{C}(\tau, a)$ son los coeficientes obtenidos después de la transformación en el domino Wavelet.

Para poder obtener la respuesta en frecuencia con el transformador en servicio usando la Transformada Wavelet se hace necesario calcular el valor RMS de los coeficientes obtenidos en el dominio de la escala (dominio Wavelet), usando la ecuación (3) [28-33].

$$
\left|Z_{a}\right|=\sqrt{\frac{\int\left|C W T_{v(t)}(\tau, a)\right|^{2} d \tau}{\int\left|C W T_{i(t)}(\tau, a)\right|^{2} d \tau}}, f_{a}=\frac{f_{c}}{a}
$$

Donde:

$\tau$ : Translación de la Wavelet madre.

$a$ : Escala o dilatación de la Wavelet madre.

$\mathrm{Z}_{a}$ : Impedancia en el dominio de la escala.

$f_{a}$ : Frecuencia asociada al dominio de la escala.

$f_{c}$ : Frecuencia central de la Wavelet madre. 


\section{Etapa 3: Análisis de la información}

Una vez procesadas las señales transitorias se obtiene la impedancia del transformador on-line en función de la frecuencia tal como se indican en (4).

$$
\mathrm{Z}(\mathrm{w})=\frac{V(w)}{I(w)}
$$

Se pueden analizar diferentes casos para las medidas on-line, de acuerdo con la ubicación de los equipos de medida, el equipo de inyección y el estado de funcionamiento de los devanados del transformador. Durante la ejecución de las pruebas on-line se analizan las siguientes configuraciones:

Energizado (On-line). En este caso el transformador se alimenta por uno de sus devanados a tensión nominal mientras el otro permanece abierto ( $\sin$ carga), de esta forma se inyectan pulsos en presencia de la onda de $60 \mathrm{~Hz}$.

Con carga (On-load). El transformador se alimenta a tensión nominal por un devanado y por el otro se le conecta carga.

Las configuraciones energizado y con carga pueden presentarse durante la operación normal de un transformador en el sistema de potencia cuando se encuentra en servicio.

En las Figuras 8 y 9 se muestran las configuraciones de medida on-line y on-load, respectivamente.

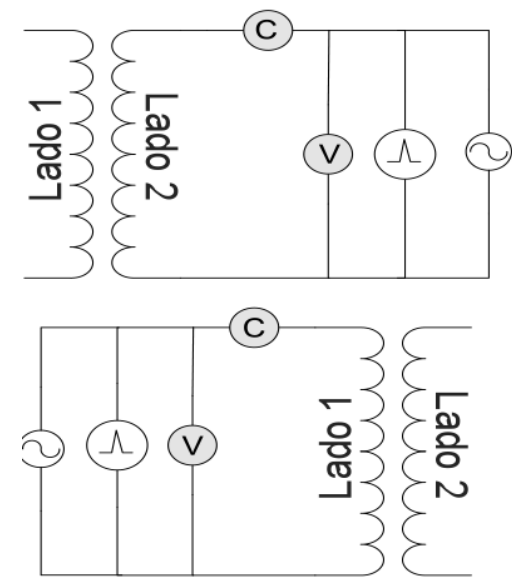

Figura 8. Configuración de medida con el transformador energizado.
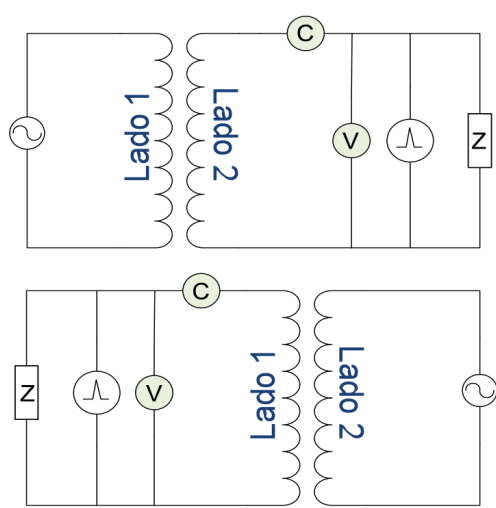

Figura 9. Configuración de medida con el transformador en carga.

El análisis de las pruebas se realizó mediante una comparación entre la Transformada de Fourier (método tradicional) y la Transformada Wavelet (método propuesto), con el fin de verificar la repetitividad de cada método, mostrando la potencialidad de la Transformada Wavelet la que se convierte en una alternativa para el procesamiento de señales transitorias para el caso FRA on-line.

En el diagrama de la Figura 10 se presenta de manera resumida el análisis de las curvas obtenidas para FRA on-line, donde se comparan el método propuesto (Transformada Wavelet) con el tradicional [33].

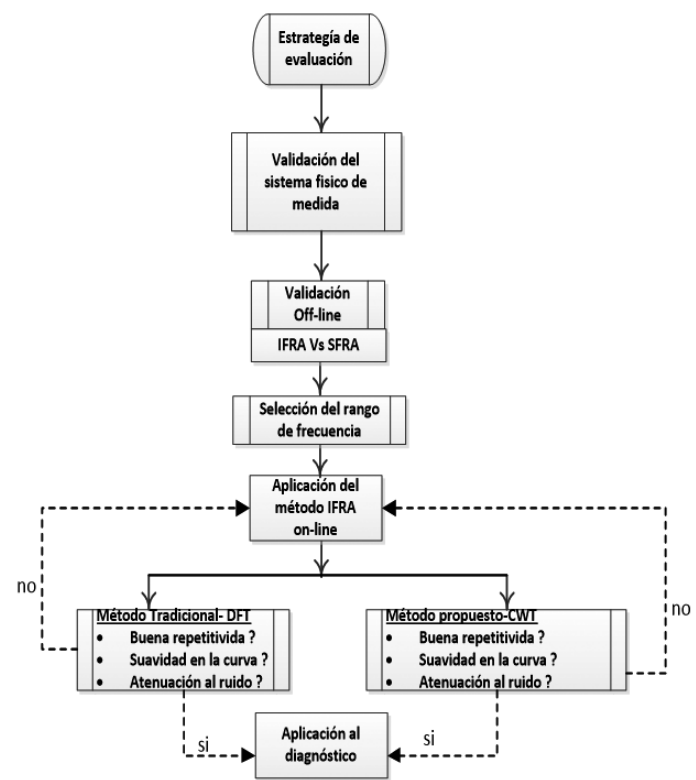

Figura 10. Diagrama de bloques a seguir para el análisis de las curvas FRA on-line. 


\section{PRUEBAS REALIZADAS EN TRANSFORMADORES REALES}

A continuación se presentan los resultados obtenidos en un transformador monofásico y uno de distribución trifásico conectados en una red eléctrica del sistema de potencia [31, 16-34].

La Figura 11 presenta el transformador de distribución monofásica (piloto) utilizado en laboratorio para pruebas de FRA on-line.

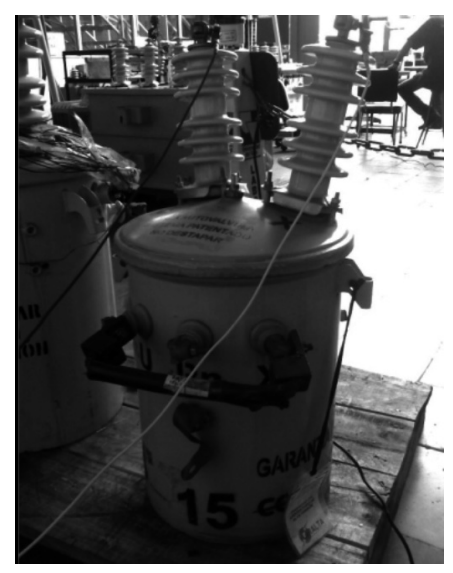

Figura 11. Transformador de distribución monofásica (piloto).

El transformador bajo prueba estaba con el lado de alta tensión energizado y el lado de baja abierto, tal como se indica en la Figura 12 con el método propuesto (CWT) y la Figura 13 con el método tradicional (DFT).

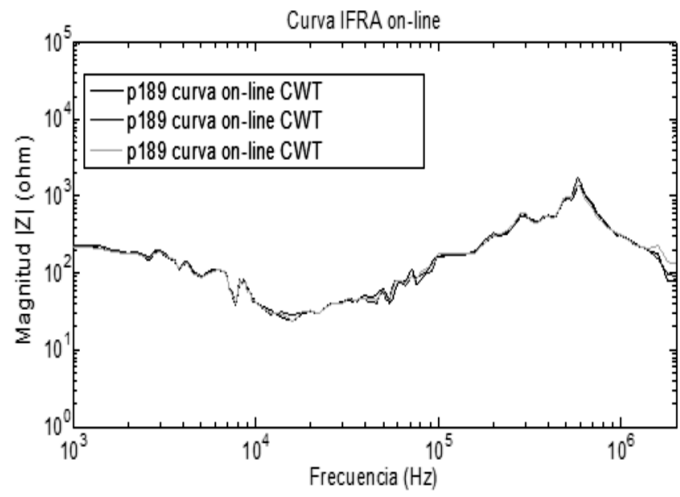

Figura 12. Respuesta on-line del transformador piloto, sin carga, usando método CWT.

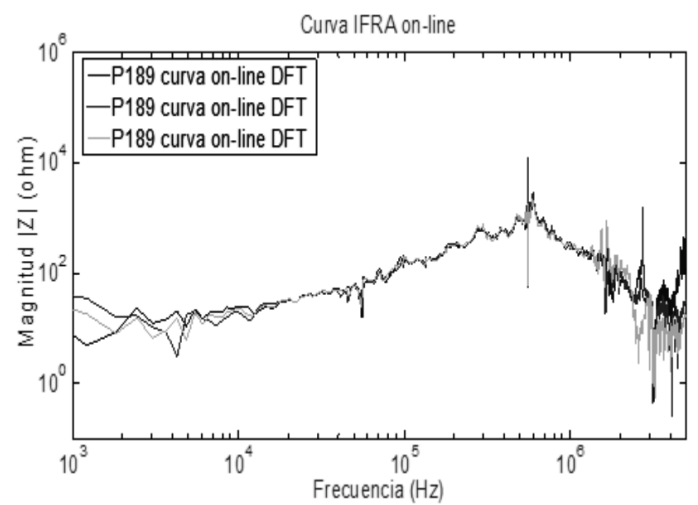

Figura 13. Respuesta on-line del transformador piloto, sin carga, usando el método DFT.

Las Figura 14 y 15 presentan la respuesta en frecuencia on-line del transformador de distribución monofásico (piloto) con el lado de alta tensión energizado y el lado de baja tensión al 50\% de carga nominal, usando el método CWT y DFT, respectivamente.

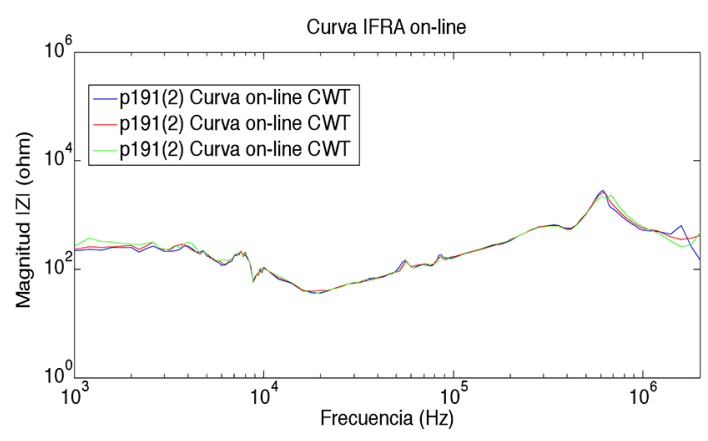

Figura 14. Respuesta on-line del transformador piloto, con carga, usando el método CWT.

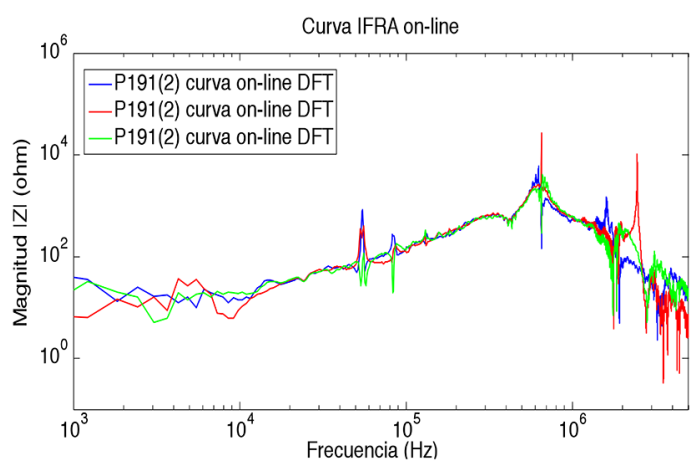

Figura 15. Respuesta on-line del transformador piloto, con carga, usando el método DFT. 
Para este caso se usaron tres pulsos controlados en distintos instantes de tiempo con el objeto de determinar la repetitividad de la prueba IFRA online usando Transformada Wavelet.

Los resultados on-line del transformador piloto en diferentes configuraciones de los devanados se presenta desde la Figura 12 hasta la Figura 15 usando el método propuesto y tradicional, el transformador se encuentra bajo una condición de no carga y carga, mostrando la aplicabilidad del método propuesto y la repetitividad en los resultados.

La Figura 16 presenta el transformador trifásico en la red eléctrica de distribución al que se le realizaron las pruebas de FRA on-line. A continuación se presentan los resultados obtenidos.

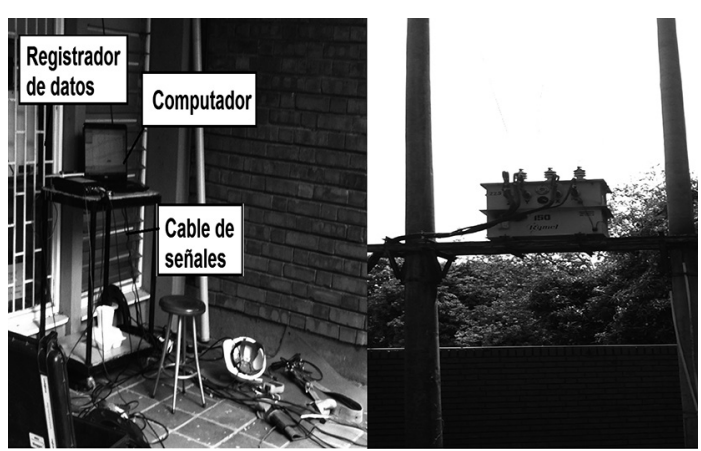

Figura 16. Transformador de distribución trifásica en la red eléctrica.

La Figura 17 presenta los resultados on-line del transformador de distribución trifásico en campo usando el método propuesto, mientras que la Figura 18 presenta los resultados usando el método tradicional, para este caso el transformador estaba energizado sin carga.

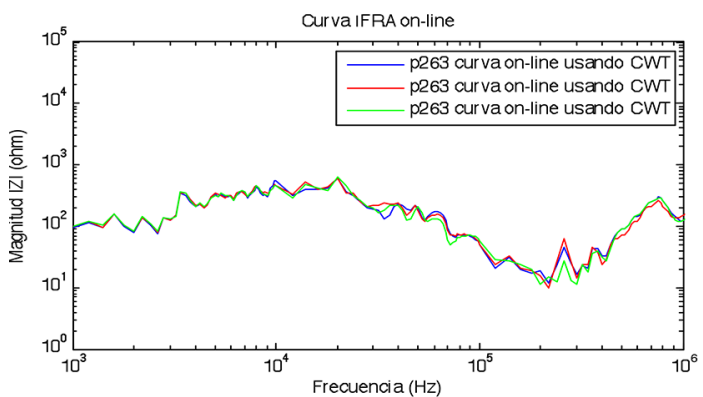

Figura 17. Respuesta on-line del transformador trifásico, sin carga, usando el método CWT.

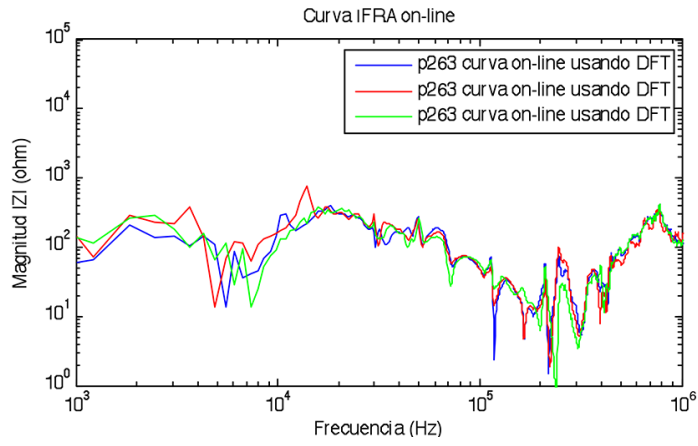

Figura 18.Respuesta on-line del transformador trifásico, sin carga, usando el método DFT.

Las Figuras 19 y 20 presentan los resultados online del transformador de distribución trifásico en campo usando el método propuesto y el método tradicional, para este caso el transformador se encontraba al 50\% de carga, por el lado de baja tensión.

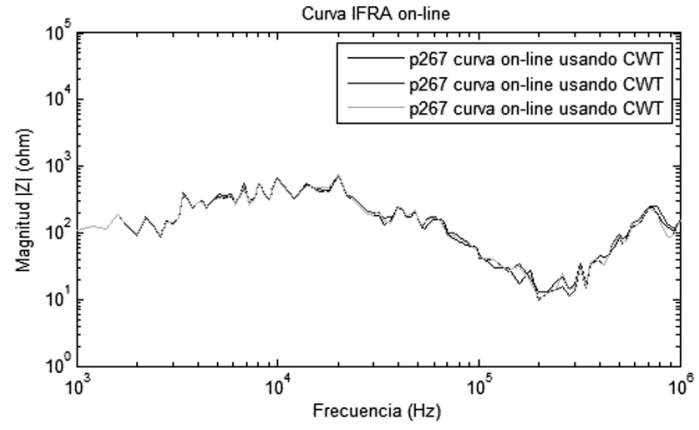

Figura 19. Respuesta on-line del transformador trifásico, con carga, usando el método CWT.

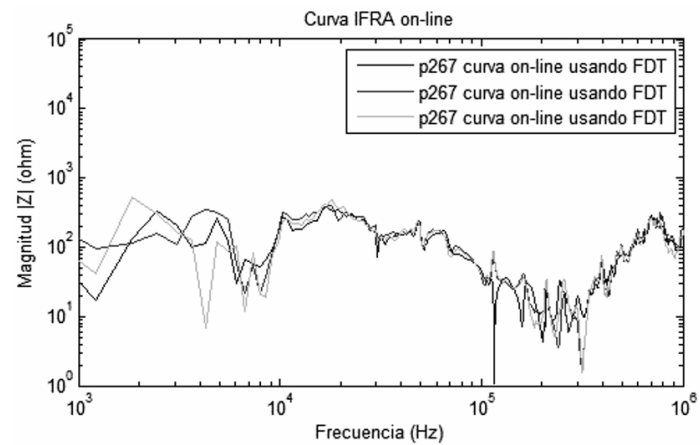

Figura 20. Respuesta on-line del transformador trifásico, con carga, usando el método DFT. 


\section{ANÁLISIS DE LOS RESULTADOS}

Al comparar las curvas on-line usando el método propuesto mediante Transformada Wavelet con las curvas mediante Transformada Discreta de Fourier (método tradicional) se observa cómo se mejora la repetitividad y eliminación de algunas resonancias presentes del tratamiento matemático con Fourier para medias y altas frecuencias tanto a nivel de laboratorio como campo, validando lo presentando en [16-29], donde se muestra que la Transformada Wavelet presenta grandes ventajas en relación con el procesamiento de la Transformada de Fourier.

Debido a que el proceso de prueba es homogéneo, es decir, es realizado bajo las mismas condiciones tanto en laboratorio como campo, se observan resultados repetibles, lo que conlleva a concluir que el método propuesto es confiable para la aplicación desarrollada en campo.

A continuación se presenta en la Tabla 1 y en la Tabla 2 los análisis cualitativo y cuantitativo, respectivamente, de los resultados obtenidos para el método FRA on-line [16].

De acuerdo con los resultados obtenidos se puede observar cómo el error relativo medio entre las curvas obtenidas es bajo cuando se usa la Transformada Wavelet Continua para el procesamiento de señal, mostrando confiabilidad en los resultados y mejorando la repetitividad.

\section{CONCLUSIONES}

Con las experiencias obtenidas de FRA online a nivel de laboratorio y campo, se brinda una alternativa para el monitoreo on-line en transformadores.

Se implementaron y validaron en campo las tres etapas propuestas en laboratorio para la medida FRA on-line, no existentes hasta el momento.

Se validó el sistema de medida e inyección de pulsos controlados para obtener la curva de respuesta en frecuencia mediante el método IFRA, evidenciando que el método propuesto es viable en campo, obteniendo buena atenuación al ruido eléctrico, suavidad en la curva y alta repetitividad.

Con el trabajo realizado se potencializó el procesamiento de señal mediante Transformada Wavelet Continua para el análisis de señales transitorias en la aplicación de la técnica FRA online mostrando mejores resultados que los obtenidos con Transformada Discreta de Fourier, en ambientes con alta incidencia de ruido.

\section{AGRADECIMIENTOS}

Los autores expresan los más sinceros agradecimientos al laboratorio de Alta Tensión de la Universidad del Valle por el apoyo y ejecución de las pruebas realizadas y al Departamento Administrativo de

Tabla 1. Análisis cualitativo de las curvas IFRA on-line.

\begin{tabular}{|l|c|c|}
\hline \multicolumn{1}{|c|}{ Parámetro analizado } & $\begin{array}{c}\text { Análisis basado en método } \\
\text { propuesto (CWT) }\end{array}$ & $\begin{array}{c}\text { Análisis basado en método } \\
\text { tradicional (DFT) }\end{array}$ \\
\hline Atenuación del ruido & Mejorado & Pobre \\
\hline Resonancias procedentes del procesamiento de señal & Mejorado & Pobre \\
\hline Repetitividad $(10 \mathrm{kHz}-1 \mathrm{MHz})$ & Mejorado & Pobre \\
\hline
\end{tabular}

Tabla 2. Análisis cuantitativo de las curvas IFRA on-line.

\begin{tabular}{|c|c|c|c|}
\hline \multirow{2}{*}{ Parámetro analizado } & $\begin{array}{c}\text { Análisis basado en método } \\
\text { propuesto (CWT) } \\
(\mathbf{\%})\end{array}$ & $\begin{array}{c}\text { Análisis basado en método } \\
\text { tradicional (DFT) } \\
(\mathbf{\% )}\end{array}$ & $\begin{array}{c}\text { Pruebas } \\
\text { realizadas }\end{array}$ \\
\hline \multirow{3}{*}{$\begin{array}{l}\text { Repetitividad: Error relativo } \\
\text { Medio (ERM) entre las respuestas } \\
\text { obtenidas con FRA on-line }\end{array}$} & 7,18 & 47,82 & $\mathrm{P} 189$ \\
\cline { 2 - 4 } & 7,10 & 54,56 & $\mathrm{P} 191$ \\
\cline { 2 - 4 } & 15,77 & 71,66 & $\mathrm{P} 263$ \\
\hline
\end{tabular}


Ciencia, Tecnología e Innovación COLCIENCIA, por el apoyo financiero durante los estudios de doctorado.

\section{REFERENCIAS}

[1] IEEE Standard C57.149. "Guide for the Application and Interpretation of Frequency Response Analysis for Oil Immersed Transformers". IEEE. March, 2013.

[2] IEC Standard 60076-18. "International Standard: Power transformers. Part 18: Measurement of Frequency Response International Electrotechnical Commission". Edition 1. July, 2012.

[3] M. Wang, A.J. Vandermaar, and K.D. Srivastava. "Improved detection of power transformer winding movement by extending the FRA high frequency range". IEEE Transactions on Power Delivery. Vol. 20 No 3, pp. 1930-1938. July, 2005.

[4] G. Aponte. "Evaluación de la condición mecánica de los transformadores mediante el análisis de su respuesta en frecuencia”. Tesis Doctoral. Escuela en Ingeniería Eléctrica y Electrónica. Universidad del Valle. Cali, Colombia. 2011.

[5] J. Pleite. "Herramienta de Modelado para el Mantenimiento Predictivo de Transformadores basado en el Análisis de la Respuesta en Frecuencia". Tesis Doctoral. Departamento de Tecnología Electrónica. Universidad Carlos III de Madrid. Madrid, España. Diciembre 2000.

[6] G.C. González. "Procedimiento de modelado basado en el análisis de la respuesta en frecuencia y aplicación en transformadores trifásicos de potencia para su caracterización y diagnostico". Tesis Doctoral. Departamento de Tecnología Electrónica, Universidad Carlos III de Madrid. Madrid, España. Enero 2012.

[7] J.A. Martínez and B.A. Mork. "Transformer Modeling for Low- and Mid-Frequency Transients-A Review". IEEE Transactions on Power Delivery. Vol. 20 Nº 2. April, 2002.

[8] G. Aponte, H. Cadavid, J. Burgos and E. Gómez-Luna. "A methodology for obtaining by measurements the transformer physicalcircuital model parameters". Electrical and Review Poland. Vol. 9a, pp. 12-15. 2012.
[9] C. Bengtsson. "Status and trends in transformer monitoring". IEEE Transactions on power delivery. Vol. $11 \mathrm{~N}^{\circ} 3$, pp. 13791384. July, 1995.

[10] T. Anderson and A. Golder. "Validation of online Power System Dynamics Measurements". IEEE Power Engineering Society Summer Meeting. Conference Proceedings Scotland, UK, pp. 1-4. 1999.

[11] T. Krieg and M. Napolitano. "Techniques and experience in on-line transformer condition monitoring and fault diagnosis in ElectraNet SA". International Conference on Power System Technology. Proceedings, pp. 10191024. 2000.

[12] C. Gonzalez, J. Pleite, V. Valdivia and J. Sanz. "An overview of the On Line Application of Frequency Response Analysis (FRA)". IEEE International Symposium on Industrial Electronics, pp. 1294-1299. 2007.

[13] E. Gómez-Luna, G. Aponte, C. GonzálezGarcía and J. Pleite. "Current Status and Future Trends in the Frequency Response Analysis (FRA) with the Transformer in Service". IEEE Transactions on Power Delivery. Vol. $28 \mathrm{~N}^{\circ} 2$, pp. 1024-1031. April, 2013.

[14] The Electric Power Industry Standard of People's Republic of China. "Frequency Response Analysis on Winding Deformation of Power Transformers". Document 151822005. 2005.

[15] CIGRE. Brochure 342. "Mechanical condition assessment of transformer Windings using Frequency Response Analysis (FRA)". Working Group A2.26. April, 2008.

[16] E. Gómez-Luna, G. Aponte and J. Pleite. "Application of Wavelet Transform to obtain the Frequency Response of a Transformer from Transient Signals - Part 2: Practical assessment and validation". IEEE Transactions on Power Delivery. Vol. 29 No 5, pp. 2231-2238. October, 2014.

[17] M.B. Figueiredo, A.D. Almeida and B. Ribeiro. "Wavelet Decomposition and Singular Spectrum Analysis for Electrical Signal Denoising". Engineering, pp. 3329-3334. 2011.

[18] V.L. Pham and K. P Wong. "Wavelettransform-based algorithm for harmonic analysis of power system waveforms". IEEE Proceedings Generation, Transmission and Distribution, pp. 249-254. 1999. 
[19] C. Duarte, P. Delmar, K. Goossen, K. Barner and E. Gómez-Luna. "Non-Intrusive Load Monitoring Based on Switching Voltage Transients and Wavelet Transforms". International Workshop Future of Instrumentation (FIIW). USA, pp. 1-4. October, 2012.

[20] F. Santamaría, C.A. Cortes and F.R. Campos. "Uso de la Transformada de Ondaletas (Wavelet Transform) en la Reducción de Ruidos en las Señales de Campo Eléctrico producidas por los Rayos". Información Tecnológica. Vol. $23 \mathrm{~N}^{\mathrm{o}}$ 1, pp. 65-78. 2012.

[21] C. Cortes, F. Santamaría, F. Roman, F. Rachidi and C. Gomes. "Analysis of Wavelet based denoising methods applied to measured lightning electric fields". 30th International Conference on Lightning Protection (ICLP). Cagliari, Italy. September 13-17, 2010.

[22] M. Misiti, Y. Misiti, G. Oppenheim and J.M. Poggi. “Wavelet Toolbox ${ }^{\mathrm{TM}}$ 4". user's Guide. Mathworks@. 2010.

[23] S. Mallat. "A wavelet tour of signal processing". Editorial Elsevier. 3ra edición. 2009.

[24] K.L. Butler and M. Bagriyanik. "Characterization of transients in transformers using discrete Wavelet Transformer". IEEE Transactions on Power Systems. Vol. 18 $\mathrm{N}^{\circ}$ 2, pp. 648-656. Mayo 2003.

[25] E. Zheng, Z. Liu and L. Ma. "Study on Harmonic Detection Method Based on FFT and Wavelet Transform". 2nd International Conference on Signal Processing Systems, (ICSPS). IEEE. Vol. 3, pp. 1-4. 2010.

[26] M.B. Figueiredo, A. de Almeida and B. Ribeiro. "Smart home: Anovel model for denoising an electrical signal". 11th International Conference on Intelligent Systems Design and Applications (ISDA), pp. 784-789. November, 2011.

[27] P.V. Kanaka, B.J. Krishna, A. Bhoomaiah, B. Singh, S. Chandra and P.A. Naidu. "Denoising of the neutral current using wavelet for diagnosis of the transformer". Electrical Insulation Conference and Electrical Manufacturing. Expo, pp. 9-12. October, 2007.

[28] E. Gómez-Luna, D. Silva, G. Aponte, J. Pleite and D. Hinestroza. "Obtaining the electrical impedance using wavelet transform from the time response". IEEE Transactions on Power Delivery. Vol. 28 $\mathrm{N}^{\circ}$ 2, pp. 1242-1244. April, 2013.

[29] E. Gómez-Luna, G. Aponte, J. Pleite, D. Silva and D. Hinestroza. "Application of Wavelet Transform to obtain the Frequency Response of a Transformer from Transient Signals - Part 1: Theoretical Analysis". IEEE Transactions on Power Delivery. Vol. 28 № 3, pp. 1709-1714. July, 2013.

[30] E. Gómez-Luna, D. Silva and G. Aponte. "Selection of a mother Wavelet for frequency analysis of transient electrical signals using WPD”. Ingeniare. Revista chilena de ingeniería. Vol. $21 \mathrm{~N}^{\circ}$ 2. July, 2013.

[31] E. Gómez-Luna, G. Aponte, W. Herrera and J. Pleite. "Experimentally obtaining on-line FRA in transformers by injecting controlled pulses". Revista Ingeniería e investigación. Vol. $33 \mathrm{~N}^{\circ}$ 1, pp. 41-45. April, 2013.

[32] E. Gómez-Luna, G. Aponte and J. Pleite. "Sistema de obtención de la respuesta en frecuencia de máquinas eléctricas”. Madrid España. Patente: ES-2534954_B1. 9 de febrero de 2016.

[33] E. Luna-Gómez. "Obtención de la Respuesta en Frecuencia de Transformadores en servicio a partir de la medida de Señales Transitorias". Tesis Doctoral. Escuela en Ingeniería Eléctrica y Electrónica. Universidad del Valle. Cali, Colombia. 2013.

[34] Grupo de Investigación en Alta Tensión GRALTA. "Desarrollo de una prueba piloto para obtener la respuesta en frecuencia de un transformador en servicio". Reporte Técnico $\mathrm{N}^{\circ}$ 2653. Escuela de Ingeniería Eléctrica y Electrónica Universidad del Valle. Agosto, 2012. 